\title{
ARTICLE Effects of COMT genotype and tolcapone on lapses of sustained attention after sleep deprivation in healthy young
}

\section{men}

Amandine Valomon ${ }^{1,2}$, Sebastian C. Holst $\mathbb{D}^{1,2}$, Alessandro Borrello ${ }^{1}$, Susanne Weigend ${ }^{1,2}$, Thomas Müller ${ }^{1}$, Wolfgang Berger ${ }^{3}$, Michael Sommerauer ${ }^{4}$, Christian R. Baumann ${ }^{2,4}$ and Hans-Peter Landolt (iD ${ }^{1,2}$

Tolcapone, a brain penetrant selective inhibitor of catechol-O-methyltransferase (COMT) devoid of psychostimulant properties, improves cognition and cortical information processing in rested volunteers, depending on the genotype of the functional Val158Met polymorphism of COMT. The impact of this common genetic variant on behavioral and neurophysiological markers of increased sleep need after sleep loss is controversial. Here we investigated the potential usefulness of tolcapone to mitigate consequences of sleep deprivation on lapses of sustained attention, and tested the hypothesis that dopamine signaling in the prefrontal cortex (PFC) causally contributes to neurobehavioral and neurophysiological markers of sleep homeostasis in humans. We first quantified in 73 young male volunteers the impact of COMT genotype on the evolution of attentional lapses during $40 \mathrm{~h}$ of extended wakefulness. Subsequently, we tested in an independent group of 30 young men whether selective inhibition of COMT activity with tolcapone counteracts attentional and neurophysiological markers of elevated sleep need in a genotype-dependent manner. Neither COMT genotype nor tolcapone affected brain electrical activity in wakefulness and sleep. By contrast, COMT genotype and tolcapone modulated the sleep loss-induced impairment of vigilant attention. More specifically, Val/Met heterozygotes produced twice as many lapses after a night without sleep than Met/Met homozygotes. Unexpectedly, tolcapone further deteriorated the sleep loss-induced performance deficits when compared to placebo, particularly in Val/Met and Met/Met genotypes. The findings suggest that PFC dopaminergic tone regulates sustained attention after sleep loss according to an inverse U-shape relationship, independently of neurophysiological markers of elevated sleep need.

Neuropsychopharmacology (2018) 43:1599-1607; https://doi.org/10.1038/s41386-018-0018-8

\section{INTRODUCTION}

Elevated sleepiness and impaired vigilance due to medical disorders, shift work, or jetlag are highly prevalent in society. Despite the risk of adverse effects, abuse, and dependence, modafinil and psychostimulant medications are frequently used to treat pathological sleepiness in patients suffering from narcolepsy, attention-deficit-hyperactivity disorder, circadian sleep-wake rhythm disorders, or behaviorally induced insufficient sleep [1]. Furthermore, pronounced inter-individual variation exists in the response to psychostimulants in sleep-disordered or sleepdeprived individuals. It was previously proposed that functional variation in the catechol-O-methyltransferase (COMT) gene may contribute to these differences [2,3], yet evidence for a causal genotype-phenotype association is currently lacking.

Prefrontal cortex (PFC) dopaminergic neurotransmission contributes to a wide range of cognitive and neuropsychiatric phenotypes [4, 5], and the COMT gene encodes the main enzyme degrading catecholamines in the PFC [6]. A common valine-tomethionine substitution at codon 158 of COMT protein (Val158Met single-nucleotide polymorphism (SNP); SNP-Id: rs4680) drastically reduces enzymatic activity [7], leading to elevated PFC dopaminergic tone in Met/Met homozygotes when compared to Val/Val homozygotes [5]. While COMT genotype modulates PFC functioning $[5,8]$, some effects of this polymorphism may only become prominent when the dopamine system is challenged, such as with behavioral or pharmacological interventions including sleep deprivation (SD) $[3,9]$ and administration of the brain penetrant COMT inhibitor tolcapone [10-12]. Tolcapone is a nonstimulant drug thought to lead to relatively specific increases in PFC dopamine $[10,11,13]$.

Because it hardly affects striatal dopaminergic transmission, tolcapone may mitigate subjective and behavioral consequences of SD without unwanted psychostimulant adverse effects. In addition, it offers the unique opportunity to dissect distinct roles of dopaminergic circuits in sleep-wake regulation. The impact of mesocortical dopaminergic neurotransmission for sleep-wake phenotypes in humans is controversial [14]. More specifically, by studying dopaminergic mechanisms of sustained attention after $\mathrm{SD}$, the wake-promoting agent modafinil was virtually ineffective in Met/Met homozygotes of COMT [3]. By contrast, in homozygous $\mathrm{Val} / \mathrm{Val}$ allele carriers, modafinil maintained optimal performance on the psychomotor vigilance test (PVT) throughout $40 \mathrm{~h}$ of prolonged wakefulness [3]. These results were compatible with a hypothesized inverse $U$-shaped relationship in the response of the

\footnotetext{
${ }^{1}$ Institute of Pharmacology and Toxicology, University of Zürich, Zürich, Switzerland; ${ }^{2}$ Zürich Center of interdisciplinary Sleep Research, University of Zürich, Zürich, Switzerland; ${ }^{3}$ Institute of Medical Molecular Genetics, University of Zürich, Zürich, Switzerland and ${ }^{4}$ Department of Neurology, University Hospital Zürich, Zürich, Switzerland Correspondence: H-P. Landolt (landolt@pharma.uzh.ch)
}

Received: 9 August 2017 Revised: 15 January 2018 Accepted: 20 January 2018

Published online: 5 February 2018 
PFC to elevated dopaminergic neurotransmission [15]. Intriguingly, neither COMT genotype nor modafinil altered the wakinginduced rebound in electroencephalographic (EEG) slow-wave activity (SWA, spectral power within the $0.75-4.5 \mathrm{~Hz}$ range) in nonrapid-eye-movement (NREM) sleep [3, 16]. In contrast to this observation, another study suggested that the Val158Met polymorphism of COMT could serve as a genetic biomarker for predicting individual differences in sleep physiology [17]. Reconciling this discrepancy is important because EEG SWA is the best established neurophysiologic biomarker of the homeostatic facet of sleep-wake regulation [18].

Here we aimed at clarifying the role of COMT in sleep-wake regulation and at testing the causality of the suggested genotype-phenotype associations. We first investigated the impact of COMT genotype on sustained attention after one night without sleep in 73 male volunteers. Subsequently, we examined the interaction of COMT genotype, tolcapone, and SD on PVT performance and neurophysiological markers of sleep-wake regulation in 30 young men. Given their baseline differences in COMT enzymatic activity, we hypothesized that sleep loss would differently impair sustained attention in Val/Val, Val/Met, and Met/ Met allele carriers, and that Val/Val homozygotes would benefit from tolcapone because it counteracts the overactive COMT and associated deficit in PFC dopaminergic tone. Our results show that tolcapone had no beneficial effect in any genotype, but further deteriorated attentional lapses after SD in Val/Met and Met/ Met allele carriers without affecting EEG markers of sleep homeostasis. The data are consistent with an inverted U-shape relationship between mesocortical dopaminergic signaling and sustained attention after sleep loss.

\section{MATERIALS AND METHODS}

\section{SD study}

Participants and genotyping. The analysis of sustained vigilant attention during extended wakefulness relied on the data of 52 male participants of three previously reported SD studies [19, 20], as well as 21 additional male volunteers completing an analogous protocol. Female subjects were excluded because COMT gene expression is modulated by estrogen-regulating sequences [21] and interactions between sex and genotype were previously reported in humans and mice $[22,23]$. No study participant worked shifts or at night, traveled more than two time zones in the past 3 months, nor suffered from any known disorder of the nervous system or an acute medical condition. Participants were free of medication and recreational drug use and consumption of excessive amounts of alcohol ( $<5$ "drinks" per week) or caffeine
( $<3$ cups of coffee or equivalent per day). They were enrolled into the studies when a pre-study screening night in the laboratory confirmed the absence of any sleep disorder, and sleep efficiency defined as the percentage of polysomnographically recorded total sleep time per time in bed (i.e., elapsed time between "lights off" and "lights on") in the screening night equaled $>80 \%$. According to the principles in the Declaration of Helsinki, all study protocols were approved by the ethics committee of the Canton of Zürich for human research. Written informed consent was obtained from all volunteers, who received a financial compensation for their participation.

In the 52 participants of the previous studies, genomic DNA was extracted from $3 \mathrm{ml}$ fresh EDTA-blood (Wizard Genomic DNA Purification Kit, Promega, Madison, WI, USA). The Val158Met polymorphism of COMT was determined using Taqman SNP Genotyping Assays (Life Technologies Europe BV). Allelic discrimination analysis was performed with SDS v2.2.2 software (Applied Biosystems, Foster City, CA, USA). In the 21 additional participants, genomic DNA was extracted from $2 \mathrm{ml}$ saliva samples using Oragene DNA kit (DNA Genotek Inc., Ottawa, Canada). The Val158Met genotypes of COMT were determined by PCR followed by sequencing. Primers used for amplification on MJ Research PTC-225 thermal cyclers (MJ Research/Bio-Rad, Reno, USA) were 5'-GATCCAAGTTCCCCTCTCTC-3' and 5'-GGTGGTCCACAAGTGTGGT$3^{\prime}$. The amplified DNA sequences were then sequenced using the Sanger chain-termination method. A Sephadex G-50 purification step was applied according to the manufacturer's instructions. The capillary gel electrophoresis and laser detection of the purified DNA products (351 bp) was performed with an ABI PRISM 3100 or ABI PRISM 3730 Genetic Analyzer (Life Technologies, Zug, Switzerland). The DNA reads from the sequencer were analyzed using the Sequencing Analysis 5.2 and SeqScape version 2.5 (both Life Technologies, Zug $\mathrm{CH}$ ) softwares. All genotypes were replicated at least once for independent confirmation.

The demographic characteristics of the participants in the "SD study" are summarized in Table 1. The $\mathrm{Val} / \mathrm{Val}$, Val/Met, and Met/ Met genotype groups did not differ with respect to age, body mass index (BMI), habitual alcohol and caffeine consumption, chronotype, subjective daytime sleepiness, and anxiety.

Study protocol. After 8-h adaptation and baseline nights (00:00-08:00), subjects were kept awake for $40 \mathrm{~h}$, and given a 10-h sleep opportunity for recovery. During prolonged wakefulness, subjects were constantly supervised and completed questionnaires, cognitive and neurobehavioral tasks, and waking EEG recordings at regular time intervals. All participants abstained from caffeine during 2 weeks prior to the start of the experiments,

Table 1. Demographic characteristics of participants in "SD" study

\begin{tabular}{|c|c|c|c|c|c|}
\hline Sample size & 17 & 35 & 21 & & \\
\hline Age (years) & $24.6 \pm 4.0$ & $23.8 \pm 3.1$ & $23.5 \pm 2.3$ & 0.68 & 0.58 \\
\hline Alcohol consumption (drinks per week) & $2.4 \pm 2.6$ & $3.6 \pm 2.8$ & $3.3 \pm 3.5$ & 0.87 & 0.42 \\
\hline Caffeine consumption (mg per day) & $147 \pm 186$ & $121 \pm 119$ & $148 \pm 109$ & 0.35 & 0.7 \\
\hline Trait Anxiety Inventory & $36.1 \pm 9.6$ & $33.9 \pm 7.8$ & $34.3 \pm 6.8$ & 0.46 & 0.63 \\
\hline
\end{tabular}


Table 2. Demographic characteristics of participants in "SD\&T" study

\begin{tabular}{|c|c|c|c|c|c|}
\hline & Val/Val & Val/Met & Met/Met & $F_{2,27}$ & $p$ \\
\hline Age (years) & $23.7 \pm 2.4$ & $23.3 \pm 1.6$ & $23.3 \pm 2.0$ & 0.11 & 0.89 \\
\hline Alcohol consumption (drinks per week) & $1.8 \pm 1.6$ & $2.9 \pm 2.8$ & $2.7 \pm 2.0$ & 0.71 & 0.50 \\
\hline Caffeine consumption (mg per day) & $110 \pm 112$ & $66 \pm 47$ & $134 \pm 106$ & 1.38 & 0.23 \\
\hline Munich ChronoType Questionnaire & $4.0 \pm 0.9$ & $4.2 \pm 1.2$ & $4.4 \pm 1.2$ & 0.38 & 0.69 \\
\hline Trait Anxiety Inventory & $32.9 \pm 9.2$ & $32.8 \pm 7.6$ & $33.1 \pm 8.6$ & 0 & 1 \\
\hline BDI-II & $5.7 \pm 4.7$ & $4.1 \pm 3.7$ & $3.9 \pm 4.7$ & 0.5 & 0.61 \\
\hline Extraversion (EPQ) & $6.5 \pm 3.6$ & $8.6 \pm 2.7$ & $7.8 \pm 3.4$ & 1.07 & 0.36 \\
\hline Neuroticism (EPQ) & $2.8 \pm 2.6$ & $1.9 \pm 1.9$ & $3.2 \pm 2.4$ & 0.82 & 0.45 \\
\hline
\end{tabular}

and from alcohol and daytime naps during at least 3 days before the studies. They were required to spend $8 \mathrm{~h}$ in bed (00:00-08:00) on the three nights before each study block. Compliance with these instructions was verified by actigraphy and sleep-wake diaries, as well as determination of saliva caffeine and breath alcohol levels upon arrival in the laboratory for sleep recordings. All studies were completed in the sleep lab of the Institute of Pharmacology and Toxicology at the University of Zürich.

Psychomotor vigilance test. A computerized version of the PVT (ePrime software, Psychology Software Tools Inc., Pittsburgh, PA, USA) was used to assess sustained vigilant attention [24]. Each test consisted of 100 trials (lasting approximately $10 \mathrm{~min}$ ), in which a numeric count-up timer appeared at random intervals on the computer screen. Participants had to press the "space" key as soon as possible upon detection of the stimulus. They completed a training session before the first adaptation night. Lapses of attention (reaction times (RTs) $>500 \mathrm{~ms}$ ), "errors of commission" $(\mathrm{RT}<100 \mathrm{~ms}$,) and "correct" responses $(100 \mathrm{~ms}<\mathrm{RT}<500 \mathrm{~ms})$ were analyzed. Speed, defined as the reciprocal values of RT, was calculated for correct responses only. Outliers were defined as values below and above 2 standard deviations of the mean, and incomplete sessions ( $<100$ trials) were excluded from analysis. To assess "time-on-task" (TOT)-dependent behavior, the task was subdivided into 5 equal quintiles, each containing 20 trials.

SD and tolcapone study

Participants and genotyping. To test causality in the observed COMT genotype-phenotype association, 30 participants were enrolled for a separate study (SD and tolcapone "SD\&T study"; see Supplementary Fig. S1). The study protocol was approved by the ethics committee of the Canton of Zürich for human research, as well as the Swiss Agency for Therapeutic Products. Written informed consent was obtained from all participants who received financial compensation for their participation. The Phase I Clinical Trial was registered on www.clinicaltrials.gov (identifier: NCT02080715). Data collection was completed between August 2013 and April 2014.

The DNA for genotyping was isolated from $200 \mu$ blood using NucleoSpin Blood (Macherey Nagel GmbH Co. KG). The Val158Met polymorphism of COMT was determined by PCR followed by sequencing as described above.

The Val/Val, Val/Met, and Met/Met genotype groups did not differ with respect to age, BMI, habitual alcohol and caffeine consumption, chronotype, subjective daytime sleepiness, and sleep quality, as well as neuropsychiatric personality traits (Table 2).

Study protocol. Study protocol and testing of performance on the PVT during prolonged waking were the same as described above. In addition, mood, tiredness, and sleepiness were quantified at $3-\mathrm{h}$ intervals with the following validated questionnaires: Profile of Mood States (fatigue, vigor, irritability, depression) [25]; Visual Analog Scales (mood, energy, motivation, and excitation); Tiredness Symptom Scale [26] and Karolinska Sleepiness Scale [27]. A morning questionnaire was administered shortly after each night to rate subjective sleep quality, including number of awakenings, total duration of time spent awake, subjective sleep latency, sleep intensity, and mood upon awakening.

Waking EEG recordings. In each study block, participants also completed at $3-\mathrm{h}$ intervals 14 wake EEG recordings, the first initiated $15 \mathrm{~min}$ after "lights on" after the baseline nights. Recordings consisted of a 3-min period with eyes closed and a 5-min period with eyes open, while sitting immobile and fixating a dot on the wall. Subjects were alerted through the intercom whenever signs of drowsiness were detected (e.g., reduced EEG alpha activity or rolling eyes). Continuous EEG, bipolar electrooculogram (EOG), electromyogram (EMG), and electrocardiogram (ECG) were recorded with Rembrandt Datalab (version 8; Embla Systems, Broomfield, CO, USA) and the polygraphic amplifier Artisan (Micromed, Mogliano Veneto, Italy). Analog signals were conditioned by a high-pass filter (EEG: $-3 \mathrm{~dB}$ at $0.15 \mathrm{~Hz}$; EMG: 10 $\mathrm{Hz}$; ECG: $1 \mathrm{~Hz}$ ) and an antialiasing low-pass filter $(-3 \mathrm{~dB}$ at $67.2 \mathrm{~Hz})$, digitized and stored with a resolution of $256 \mathrm{~Hz}$ (sampling frequency of $256 \mathrm{~Hz}$ ). Ten bipolar EEG derivations along the right and left anteroposterior axes were recorded. The data of the C3A2 derivation are reported here; artifacts were visually identified. Power spectra (Fast Fourier Transform, Hanning window) of 
A

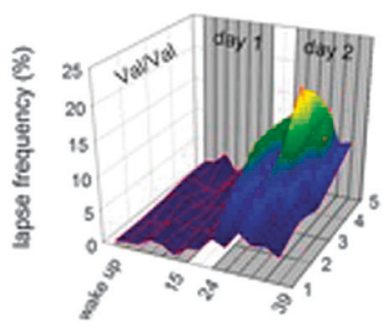

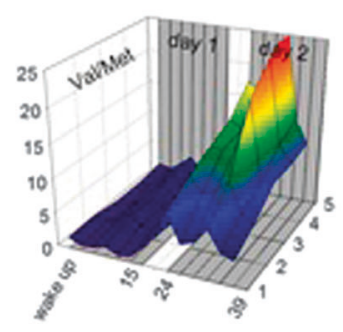

time arwake $(\mathrm{h})$
B
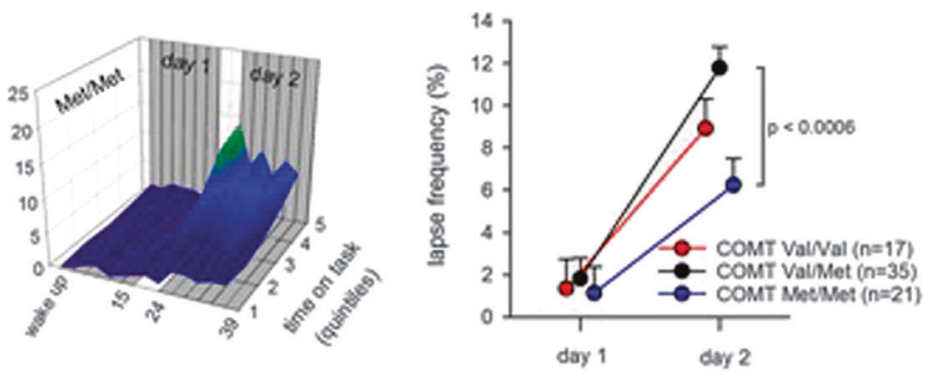

time awake (days)

Fig. 1 Lapses of attention (i.e., trials on a 10-min PVT with reaction times $>500 \mathrm{~ms}$, expressed as a percentage of all trials in a quintile) across $40 \mathrm{~h}$ of sustained wakefulness, split by COMT genotypes. A PVT lapses ( $y$-axis) as a function of time on task ( $z$-axis) and time awake ( $x$-axis). The warmer the colors, the higher the lapse frequency. The 3D plots are split by COMT genotypes (left panel: Val/Val; middle panel: Val/Met; right panel: Met/Met). B Comparison of lapse frequencies in baseline (day 1) and after sleep deprivation (day 2), split by COMT genotypes (Val/Val: red line; Val/Met: black line; Met/Met: blue line). Values represent estimated means \pm standard error; $p$ value $(p<0.0006)$ withstands Bonferroni correction $(p<0.016)$

artifact-free, 50\%-overlapping 2-s epochs were computed with MATLAB ${ }^{\oplus}$ (The MathWorks Inc., Natick, MA, USA), resulting in a 0.5 $\mathrm{Hz}$ frequency resolution. Frequency bins between 1 and $20 \mathrm{~Hz}$ were analyzed. To compare the waking EEG between baseline and after SD, mean values recorded at 08:00, 11:00, 14:00, and 17:00 on the second day of prolonged wakefulness were expressed as a percentage of the respective mean values of the baseline day.

Polysomnographic recordings. Continuous recording of EEG, EOG, EMG, and ECG was performed during all nights. Standard sleep stages [28] were visually scored in 20-s epochs (C3A2 derivation) with Rembrandt Analysis Manager (version 8; Embla Systems, Broomfield, CO, USA). Movement-related and arousal-related artifacts were visually identified and eliminated. EEG spectra (FFT routine, Hanning window, $50 \%$ overlapping 4-s epochs, $0.25 \mathrm{~Hz}$ resolution) were calculated with MATLAB $^{\circ}$, averaged over 5 consecutive 4-s epochs, and matched with the sleep scores. To compute all-night power spectra in NREM sleep (stages 2, 3, and 4) and REM sleep, all artifact-free 20 -s values were averaged. NREM/ REM sleep cycles were defined according to Feinberg and Floyd [29]. Data analyses were restricted to the first $8 \mathrm{~h}$ of the recovery nights. Relative EEG power values in the recovery nights were normalized to the average all-night EEG power of the two baseline nights.

Tolcapone. Tolcapone is an orally active, reversible, competitive inhibitor of COMT. Following oral administration, the compound crosses the blood-brain barrier and has a specific effect on PFC dopamine neurotransmission [30, 31]. Two doses of $100 \mathrm{mg}$ tolcapone, in the form of capsules, were administered to all subjects after $11 \mathrm{~h}$ (at 19:00) and $23 \mathrm{~h}$ (at 07:00) of prolonged wakefulness according to a randomized, double-blind, placebocontrolled, crossover design. In vitro work suggests that at a dose of $100 \mathrm{mg}$, tolcapone induces maximum inhibition (72\%) of COMT enzymatic activity after approximately 1-1.5 h, while activity returns to baseline levels after roughly $13 \mathrm{~h}$ [32]. These pharmacodynamic properties of tolcapone permitted us to address the question whether COMT inhibition during prolonged wakefulness affects the sleep EEG independently of an acute pharmacological effect. Capsules and random allocation sequence were produced by the "Kantonsapotheke Zürich" by homogenizing commercial Tasmar Tolcaponum $100 \mathrm{mg}$ tablets (distribution: MEDA Pharma GmbH, Wangen-Brüttisellen, Switzerland) with mannitol (Siegfried Ltd, Zofingen, Switzerland); placebo capsules of identical appearance contained only mannitol. Verum and placebo were administered 1 week apart to two randomization groups, containing 5 individuals of each COMT genotypes and differing by the sequence of drug administration.
Due to the potential hepatotoxicity of tolcapone, liver functions were assessed before and after the experiment. They were required to lay within normal range for study participation (alanine transaminase: $<82 \mathrm{U} / \mathrm{l}$; aspartate transaminase: $<76 \mathrm{U} / \mathrm{l}$ ).

Statistical analyses. Performance on the PVT was assessed with the SAS 9.2 software (SAS Institute, Cary, NC, USA) similarly to previous studies [33]. Linear mixed models for repeated measures with the random effect "subject," the between-subjects factor "genotype" (Val/Val, Val/Met, Met/Met), and the within-subjects factors "day" (baseline, SD), "time" (14 sessions), "TOT" (quintiles 1-5), "treatment"(placebo, tolcapone), "condition" (baseline night, placebo recovery night, tolcapone recovery night), and "NREM sleep episode" (1-4) were conducted as specified in the text and figure legends. If not stated otherwise, estimated means and standard errors of corresponding mixed models are illustrated. Number of lapses was defined as percentage within each quintile ("lapse frequency") and absolute EEG power densities were logtransformed before statistical analyses, to approximate a normal distribution. Effect sizes (partial eta squared: $\eta_{P}{ }^{2}$ ) were calculated from corresponding analysis of variance (ANOVA) $F$ values and degrees of freedom. Effect sizes of $0.01,0.059$, and 0.138 are considered small, moderate, and large [34, 35]. Significance level was set at $a<0.05$. Bonferroni-corrected post hoc tests to correct for multiple comparisons are reported where appropriate.

\section{RESULTS}

COMT genotype contributes to individual differences in attentional lapses after SD

Prolonged wakefulness and TOT increased the number of lapses on the PVT, reflecting well-established homeostatic and circadian influences ("time": $F_{5,3720}=12.00, p<0.0001, \eta_{\mathrm{P}}{ }^{2}=0.016$; "day": $F_{1,3720}=500.9 p<0.0001, \quad \eta_{\mathrm{P}}{ }^{2}=0.119 ;$ "TOT": $F_{4,3720}=22.0, p<$ $0.0001, \eta_{P}{ }^{2}=0.023$ ) (Fig. 1A). Moreover, COMT genotype modulated the impairment in PVT performance over time ("genotype" $\mathrm{x}$ "time" interaction: $F_{10,3720}=2.47, p<0.006, \eta_{\mathrm{P}}{ }^{2}=0.007$ ). To better illustrate this main finding of the "SD study," Fig. 1B depicts mean lapse frequency on the days before and after SD. While all genotypes performed equally well without virtually any lapses in baseline, they clearly differed on the second day ("genotype" $x$ "day" interaction: $\left.F_{2,3720}=19.16, p<0.0001, \eta_{P}{ }^{2}=0.010\right)$. Specifically, Val/Met heterozygotes produced almost the double number of lapses $(11.8 \pm 1.0, n=35)$ on the day after sleep loss when compared to Met/Met homozygotes $(6.2 \pm 1.3, n=21)$ (Fig. 1B). A similar yet less striking modulation by COMT genotype was observed in the placebo condition in the 30 independent 
A

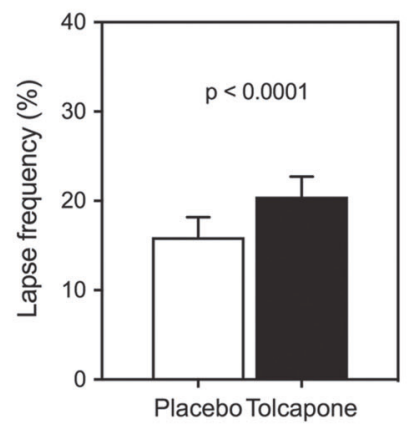

B

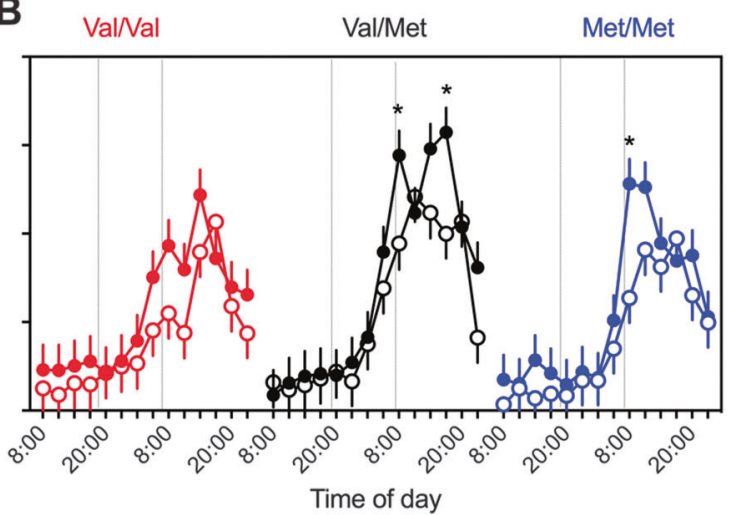

Fig. 2 The increase in attentional lapses on a 10-min PVT after sleep deprivation is exacerbated by the COMT inhibitor tolcapone and modulated by COMT genotype. A Average lapse frequencies after sleep deprivation (day 2) in placebo (white) and tolcapone (black) conditions. Bars represent means \pm SEM $(n=30)$. B Evolution of lapse frequency on the PVT, split by treatment (placebo: open circles; tolcapone: filled circles) and genotype (Val/Val: red; Val/Met: black; Met/Met: blue). Estimated means \pm standard error ( $n=10$ per group). Tolcapone administration is indicated with vertical lines. ${ }^{*} p<0.0004$ (tolcapone vs. placebo; withstanding Bonferroni correction)

participants of the "SD\&T study" ("genotype" $x$ "day" interaction: $F_{2,1524}=6.0, p<0.003, \eta_{\mathrm{P}}^{2}=0.008$ ) (see Fig. 2B).

COMT genotype and COMT inhibition have no impact on neurophysiological and behavioral markers of elevated sleep pressure

To tackle the question whether the build-up of homeostatically regulated sleep pressure is modulated by COMT genotype and selective pharmacological inhibition of COMT enzymatic activity, detailed quantitative analyses of the EEG in wakefulness and sleep were performed. On the baseline day, absolute spectral power between 1 and $20 \mathrm{~Hz}$ averaged over all waking EEG recordings from 08:00 to 17:00 was similar in all genotypes ("genotype": $F_{2,25}$ $<3.8, p_{\text {all }}>0.06$ ). SD increased EEG activity in all bins below $9 \mathrm{~Hz}$ and above $11.5 \mathrm{~Hz}$ ("condition": $F_{1,72}>3.89, p_{\text {all }}<0.05, \eta_{\mathrm{P}}{ }^{2}=0.051$ ). Figure $3 \mathrm{~A}$ illustrates the evolution of delta-theta activity $(1-9 \mathrm{~Hz}$ band) across prolonged wakefulness in $\mathrm{Val} / \mathrm{Val}, \mathrm{Val} / \mathrm{Met}$, and Met/ Met genotypes of COMT. In all genotypes, the evolution of EEG activity reflected similar homeostatic and circadian influences ("time": $F_{13,261}=20.3, p<0.0001, \eta_{P}^{2}=0.502$; "genotype": $F_{2,26}=$ $2.49, p>0.1$, which was indistinguishable between tolcapone and placebo ("treatment": $F_{1,71}=0.18, p>0.6$; "genotype" $x$ "treatment" interaction: $\left.F_{2,71}=0.69, p>0.5\right)$.

Compared to the baseline night, SD increased EEG SWA, slowwave sleep, total sleep time, and sleep efficiency in the recovery night irrespectively of COMT genotype and tolcapone treatment (Fig. 3B and Supplementary Table S1). Absolute SWA values, the dissipation of SWA across consecutive NREM sleep episodes in baseline and recovery nights, as well as the relative increase in SWA after SD were similar among genotypes and treatment conditions. More specifically, three-way mixed-model ANOVA with the factors "genotype," "condition," and "NREM sleep episode" yielded significant main effects and interaction of "condition" $\left(F_{2,295}=84.3, p<0.0001, \eta_{\mathrm{P}}{ }^{2}=0.363\right)$ and "episode" $\left(F_{3,293}=488.7\right.$, $p<0.0001, \eta_{\mathrm{P}}{ }^{2}=0.833$; "condition" $\mathrm{x}$ "episode" interaction: $F_{6,293}=$ $\left.11.8, p<0.0001, \eta_{\mathrm{P}}{ }^{2}=0.195\right)$, yet no effects involving "genotype." Furthermore, no significant main effects of "genotype" or "treatment," or interactions with the factor "condition" were observed in visually scored sleep variables and subjective ratings of sleep quality (Supplementary Table S1).

SD also increased sleepiness, fatigue, tiredness, and irritability, and reduced response speed on the PVT, vigor, energy, motivation, and mood. These typical effects of prolonged wakefulness were not modulated by COMT genotype nor tolcapone treatment (Supplementary Fig. S2).
Tolcapone enhances attentional lapses after SD depending on COMT genotype

Although they were devoid of effects on neurophysiological and state markers of elevated sleepiness after sleep loss, both COMT genotype and tolcapone affected attentional lapses after SD. Prolonged wakefulness and time-on-task enhanced lapsing also in the "SD\&T study" ("time": $F_{5,2937}=12.7, p<0.0001, \eta_{\mathrm{P}}{ }^{2}=0.021$; "day": $F_{1,2937}=768.0, p<0.0001, \eta_{\mathrm{P}}{ }^{2}=0.207$; "TOT": $F_{4,2937}=11.1, p$ $\left.<0.0001, \eta_{P}^{2}=0.015\right)$. Unexpectedly, however, rather than improving performance, tolcapone further increased lapse frequency on the day following sleep loss when compared to placebo $(20.3 \pm 1.4$ vs. $15.8 \pm 1.4$; "treatment": $F_{1,2937}=27.8, p<0.0001, \eta_{\mathrm{P}}{ }^{2}=0.009$; "treatment" $x$ "day" interaction: $F_{1,2937}=7.1, p=0.009, \eta_{\mathrm{P}}{ }^{2}=0.002$; Fig. 2a). When analyzing the data on a finer time scale, a significant "genotype" $x$ "treatment" $x$ "time" interaction was observed $\left(F_{10,2937}=3.2, p<0.0005, \eta_{\mathrm{P}}{ }^{2}=0.011\right)$. More specifically, intake of tolcapone at 07:00 after the night without sleep significantly deteriorated performance in Val/Met and Met/Met genotypes when compared to placebo $(p<0.05$; Fig. $2 b)$. The difference between the treatment conditions was insignificant in the $\mathrm{Val} / \mathrm{Val}$ genotype. By contrast, in Val/Met heterozygotes, performance between tolcapone and placebo still differed at $17: 00,10 \mathrm{~h}$ after the second tolcapone ingestion.

\section{DISCUSSION}

Because tolcapone appears to be free of major psychostimulant properties and abuse potential [13], the pharmacodynamic profile of this COMT inhibitor may be beneficial to improve vigilance in sleepy patients and healthy individuals undergoing SD. Indeed, previous research consistently concluded that this compound may improve distinct aspects of cognitive functions in Parkinson's patients [36] and in healthy volunteers [10, 11, 13], as well as in rats and mice $[12,37,38]$. While we expected that especially individuals with high COMT activity (Val/Val genotype) may benefit from pharmacological COMT inhibition, we found that tolcapone does not improve sustained attention after prolonged wakefulness in any COMT genotype. On the contrary, it increased attentional lapses in Val/Met and Met/Met allele carriers.

Sustaining focused attention while performing a 10-min PVT is a challenging task, especially when sleep deprived. Increased lapsing compared to the rested state has been assumed to reflect transient cognitive disruptions referred to as "wake-state instability" [39]. The precise neural bases of how acute total sleep loss impairs sustained attention are currently unknown [40]. 

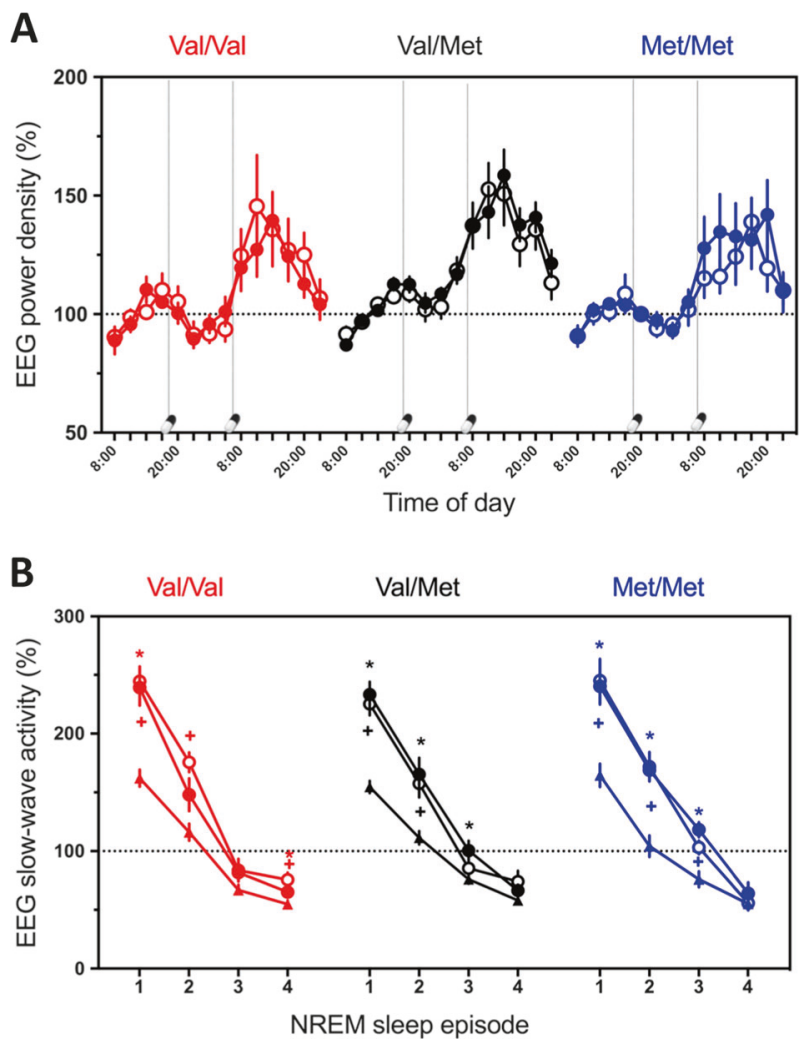

Fig. 3 The homeostatic build-up in sleep pressure is indistinguishable among COMT genotypes and not affected by tolcapone. A Evolution of $1-9 \mathrm{~Hz}$ activity in the waking EEG across $40 \mathrm{~h}$ prolonged wakefulness. EEG activity in placebo (open circles) and tolcapone (filled circles) conditions was expressed as a percentage of the mean values at 08:00, 11:00, 14:00, and 17:00 on the baseline day (dashed horizontal line), split by COMT genotypes (Val/Val: red line; Val/Met: black line; Met/Met: blue line). Administration of tolcapone at 7:00 and 07:00 is indicated with capsule and vertical dashed lines. Threeway mixed-model ANOVA with the factors "genotype," "treatment," and "time" yielded significant effects of "time," yet no significant effects and interactions with "genotype" or "treatment" (see main text). B Dynamics of mean SWA across NREM sleep episodes 1-4 in baseline (mean of two baseline nights) and recovery nights. EEG activity (means \pm SEM) in placebo (open circles) and tolcapone conditions (filled circles) was expressed as a percentage of the mean all-night values in baseline (dashed horizontal line), split by COMT genotypes (Val/Val: red line; Val/Met: black line; Met/Met: blue line). Three-way mixed-model ANOVA with the factors "genotype," "condition," and "NREM sleep episodes" yielded significant effects of "episode," "condition," and "cycle" $x$ "condition," yet no significant interactions involving "genotype" (see main text). Values represent estimated means \pm standard error. ${ }^{*} p<0.05$ (tolcapone recovery vs. mean baseline; paired, two-tailed $t$ tests). ${ }^{+} p<0.05$ (placebo recovery vs. mean baseline; paired, two-tailed ttests)

Nevertheless, a core brain network comprising dorsomedial, midventrolateral and ventrolateral PFC, anterior insula, and parietal areas, as well as subcortical structures including cerebellar vermis, thalamus, basal ganglia, and midbrain subserves vigilant attention [41]. The midbrain is the origin of three major dopaminergic pathways [42]. The nigrostriatal pathway projects from the substantia nigra to the striatum (caudate/putamen) and plays an important role in motor control. The mesolimbic pathway is associated with the reward circuit. This pathway originates in the ventral tegmental area (VTA), connects to several structures of the limbic system, and is important for memory, reward, and motivation behaviors. Similarly, the mesocortical pathway also originates in VTA, but projects to the frontal cortex and surrounding structures. Given that dopaminergic

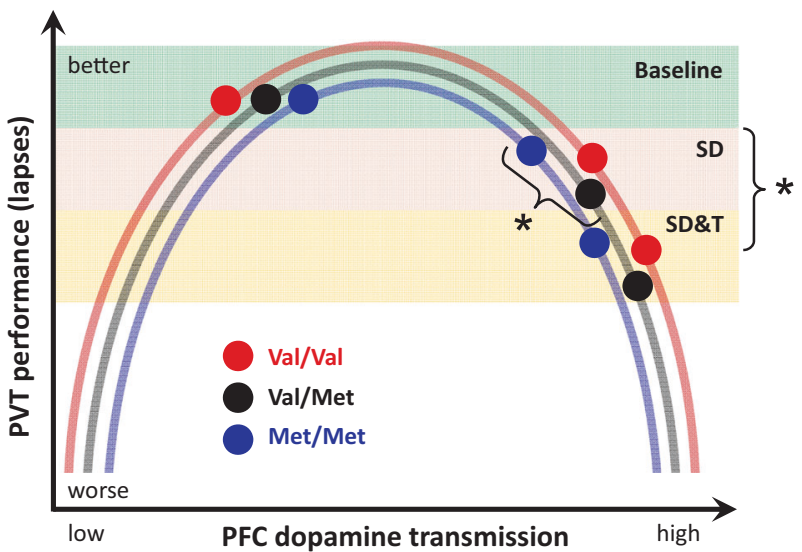

Fig. 4 Schematic illustration of the proposed relationships between PFC dopamine transmission and attentional lapses in baseline (green shading), after sleep deprivation (orange shading), and after sleep deprivation and tolcapone administration (yellow shading), split by COMT genotypes (Val/Val: red; Val/Met: black; Met/Met: blue). The data suggest an inverse U-shaped relationship between PFC dopamine transmission and lapse frequency. While the increase in PFC dopamine transmission with sleep deprivation may be relatively more pronounced in $\mathrm{Val} / \mathrm{Val}$ than in $\mathrm{Val} / \mathrm{Met}$ and Met/Met allele carriers, tolcapone reduces COMT activity to roughly the same level in all three genotypes [13]. Asterisks indicate impacts of genotype and tolcapone treatment after sleep deprivation. SD: sleep deprivation study, SD\&T: sleep deprivation and tolcapone study. See Discussion for details

neurotransmission in the mesocortical pathway is primarily controlled by COMT $[43,44]$, the present genetic and pharmacogenetic data suggest that the mesocortical dopaminergic system importantly contributes to the regulation of lapses in sustained attention after SD. Nevertheless, it needs to be kept in mind that vigilant attention during SD is regulated by several neurotransmitters, and yet unknown factors and their interactions could influence the current findings. For example, previous reports have shown that benefits of the cholinesterase inhibitor, donepezil, were most pronounced in study participants whose vigilance clearly declined in the placebo condition when sleep deprived, whereas those participants who maintained vigilance when receiving placebo worsened under donezepil $[45,46]$. Apart from increasing cholinergic neurotransmission, donepezil secondarily also influences dopaminergic and noradrenergic signaling. In addition, the interpretation of the results should consider that the COMT enzyme, which is present in the brain and the periphery, not only metabolizes dopamine but also various other compounds including the dopamine derivatives norepinephrine and epinephrine.

SD\&T modulate attentional lapses according to an inverse Ushaped relationship

It is well established that genetic variation of COMT modulates body and brain functions according to U-shaped relationships (e.g., $[11,13,15,44])$. Corroborating the view that dopaminergic agonists can correct behavioral consequences of suboptimal PFC dopaminergic signaling, metamphetamine and tolcapone reliably improved different aspects of memory, executive functioning, and risk taking in non-sleep-deprived $\mathrm{Val} / \mathrm{Val}$ allele carriers, but were ineffective or even impaired performance in Met/Met individuals $[10,11,13,15]$. The present study demonstrates that beyond and above COMT genotype, a pre-existing sleep debt strongly impacts on the effects of a pharmacological increase of dopaminergic neurotransmission on brain functions. This conclusion is consistent with a previous study, showing that a low dose of modafinil maintained optimal sustained attention, executive functioning 
and subjective well-being for as long as 2 days and 1 night of prolonged wakefulness in Val/Val homozygotes, yet was ineffective in matched Met/Met allele carriers [3]. The current work extends these findings and suggests that similar to other PFCdependent processes such as working memory and risky decision making $[4,5,10]$, there is an inverted U-shaped relationship between dopamine activity in PFC and attentional lapses, and this relationship is modulated by sleep loss.

As schematically illustrated in Fig. 4, the data suggest that lapse frequency on a 10-min PVT is optimal in well-rested study participants and indistinguishable among $\mathrm{Val} / \mathrm{Val}, \mathrm{Val} / \mathrm{Met}$, and Met/Met genotypes of COMT (also see [3, 17]). Wakefulness increases dopamine concentrations in periphery and central nervous system, including nucleus accumbens and medial PFC, in rats [47, 48], as well as in human plasma [49]. Thus, SD can be assumed to increase PFC dopamine to super-optimal levels. Due to the genotype-dependent differences in COMT enzymatic activity and associated differences in baseline dopaminergic neurotransmission, the relative increase in dopaminergic tone after prolonged waking may be most pronounced in $\mathrm{Val} / \mathrm{Val}$, intermediate in Val/Met, and weakest in Met/Met genotype, leading to the observed differences in PVT lapses after sleep loss. Instead of mitigating the consequences of sleep loss, tolcapone treatment induced even more pronounced impairment. Future studies are warranted to probe the dose-effect relationships of the interaction between total and partial SD\&T, to test whether there is an additive interaction of sleep loss and inhibition of COMT. This work will have considerable translational relevance because COMT inhibitors are used as adjunctive treatment in Parkinson's disease and have been considered for patients and people with impaired vigilance due to insufficient sleep.

Tolcapone and sleep-wake regulation

While it is well established that serotonin, histamine, and norepinephrine importantly contribute to sleep-wake regulation (for a recent review, see [2]), the exact roles of dopamine in regulating sleep remain incompletely understood. Recent studies in flies, mice, and humans accumulated convergent evidence that mesolimbic dopaminergic pathways importantly contribute to the maintenance of wakefulness, the homeostatic regulation of sleep and arousal, as well as sleep-wake-dependent behaviors [20, 33, 50-55]. By contrast, the available studies on the impact of the mesocortical pathway and COMT activity on sleep-wake regulation are inconclusive. For example, research in large samples of individuals studied under field conditions with questionnaires, actigraphy, and waking EEG revealed no consistent influence on daytime sleepiness and physiological markers of circadian and homeostatic sleep-wake regulation [56-58]. Nevertheless, a laboratory study employing a chronic partial sleep restriction protocol suggested that Met/Met homozygotes of the Val158Met polymorphism of COMT exhibit a steeper decline in EEG slowwave energy during sleep than $\mathrm{Val} / \mathrm{Met}$ and $\mathrm{Val} / \mathrm{Val}$ allele carriers [17]. This observation was interpreted to reflect a differential homeostatic response to sleep loss among different COMT genotypes. However, another well-controlled study found no differences between $\mathrm{Val} / \mathrm{Val}$ and Met/Met homozygotes in established sleep and EEG markers of sleep homeostasis in baseline and after acute total SD [3, 16]. The current investigation confirmed that functional genetic variation and selective pharmacological inhibition of COMT enzymatic activity do not affect established waking and sleep EEG markers of sleep homeostasis when challenged by acute total SD. The data, thus, indicate that the mesocortical dopaminergic system has no major role in regulating sleep homeostasis. Supporting this conclusion, the monoaminergic wake-promoting agent, modafinil, and the irreversible monoamine oxidase inhibitor, phenelzine, do not alter the dynamics of EEG SWA in NREM sleep [16, 20, 59]. The dissociation between sleep-wake-dependent changes in neurobehavioral performance, subjective sleepiness, and neurophysiologic markers of sleep pressure has been highlighted in various previous publications (e.g., [33, 39, 66, 67]), suggesting that these markers of elevated sleep drive are regulated by different underlying mechanisms.

\section{Main conclusions}

In summary, the current results demonstrate that PFC dopaminergic neurotransmission contributes to the regulation of attentional lapses during SD without changing neurophysiological markers of sleep homeostasis. Intriguingly, selective inhibition of COMT can even reverse the expected beneficial effects of pharmacologically enhanced dopaminergic tone after SD. This finding has important implications, given the increased use of stimulants in healthy people aiming at improved vigilance and cognitive functions $[1,60]$. Nevertheless, the data presented here were obtained in healthy young men and the results of this study may not be generalized to women and older individuals. Future pharmacogenetics studies are warranted to disentangle the complex roles of the dopaminergic system in regulating human sleep-wake behavior and physiology.

\section{ACKNOWLEDGEMENTS}

We thank Alexandra Sousek, Dr. Emily Urry, and lan Clark for help in data collection and supervision of participants. Drs. Roland Dürr, Thomas Rusterholz and Peter Achermann for help with data analyses. Profs. Alexander Borbély and Irene Tobler for helpful discussions. Dr. Britta Seebauer for help with the genotyping and Prof. Steven Brown for providing access to his laboratory for the isolation of DNA.

\section{FUNDING}

This was not an industry-sponsored study. This work was supported by the University Research Priority Program "Integrative Human Physiology" of the UZH, the Clinical Research Priority Program "Sleep and Health" of the UZH, the Swiss National Science Foundation (grant \# 320030_163439), the Forschungskredit of the University of Zurich (grant \# FK-15-039), and the Theodor und Ida Herzog-Egli-Stiftung.

\section{ADDITIONAL INFORMATION}

Supplementary information accompanies this paper at https://doi.org/10.1038/ s41386-018-0018-8.

Conflict of interest: The authors declare that they have no conflict of interest.

Publisher's note: Springer Nature remains neutral with regard to jurisdictional claims in published maps and institutional affiliations.

\section{REFERENCES}

1. Farah MJ. The unknowns of cognitive enhancement. Science (80-). 2015;350: 379-80.

2. Holst SC, Valomon A, Landolt H-P. Sleep pharmacogenetics: personalized sleep-wake therapy. Annu Rev Pharmacol Toxicol. 2016;56:577-603.

3. Bodenmann S, Xu S, Luhmann UFO, Arand M, Berger W, Jung $\mathrm{HH}$, et al. Pharmacogenetics of modafinil after sleep loss: catechol-O-methyltransferase genotype modulates waking functions but not recovery sleep. Clin Pharmacol Ther. 2009;85:296-304.

4. Goldman-Rakic PS, Muly EC, Williams GV. D1 receptors in prefrontal cells and circuits. Brain Res Rev. 2000;31:295-301.

5. Tunbridge EM, Harrison PJ, Weinberger DR. Catechol-O-methyltransferase, cognition, and psychosis: Val158Met and beyond. Biol Psychiatry. 2006;60:141-51.

6. Matsumoto M, Weickert CS, Akil M, Lipska BK, Hyde TM, Herman MM, et al. Catechol O-methyltransferase mRNA expression in human and rat brain: evidence for a role in cortical neuronal function. Neuroscience. 2003;116:127-37.

7. Chen J, Lipska BK, Halim N, Ma QD, Matsumoto M, Melhem S, et al. Functional analysis of genetic variation in catechol-O-methyltransferase (COMT): effects on mRNA, protein, and enzyme activity in postmortem human brain. Am J Hum Genet. 2004;75:807-21.

8. Egan MF, Goldberg TE, Kolachana BS, Callicott JH, Mazzanti CM, Straub RE, et al. Effect of COMT Val108/158 Met genotype on frontal lobe function and risk for schizophrenia. Proc Natl Acad Sci USA. 2001;98:6917-22. 
9. Satterfield BC, Hinson JM, Whitney $P$, Schmidt MA, Wison JP, van Dongen $H$. Catechol-O-methyltransferase (COMT) genotype affects cognitive control during total sleep deprivation. Cortex. 2017. https://doi.org/10.1016/j.cortex.2017.11.012.

10. Farrell SM, Tunbridge EM, Braeutigam S, Harrison PJ. COMT Val158Met genotype determines the direction of cognitive effects produced by catechol-O-methyltransferase inhibition. Biol Psychiatry. 2012;71:538-44.

11. Giakoumaki SG, Roussos P, Bitsios P. Improvement of prepulse inhibition and executive function by the COMT inhibitor tolcapone depends on COMT Val158Met polymorphism. Neuropsychopharmacology. 2008;3382:3058-68.

12. Barkus C, Korn C, Stumpenhorst K, Laatikainen LM, Ballard D, Lee S, et al. Genotype-dependent effects of COMT inhibition on cognitive function in a highly specific, novel mouse model of altered COMT activity. Neuropsychopharmacology. 2016;41:3060-9.

13. Apud JA, Mattay V, Chen J, Kolachana BS, Callicott JH, Rasetti R, et al. Tolcapone improves cognition and cortical information processing in normal human subjects. Neuropsychopharmacology. 2007;32:1011-20.

14. Dauvilliers $Y$, Tafti M, Landolt HP. Catechol-O-methyltransferase, dopamine, and sleep-wake regulation. Sleep Med Rev. 2015;22:47-53.

15. Mattay VS, Goldberg TE, Fera F, Hariri AR, Tessitore A, Egan MF, et al. Catechol Omethyltransferase val158-met genotype and individual variation in the brain response to amphetamine. Proc Natl Acad Sci USA. 2003;100:6186-91.

16. Bodenmann S, Landolt H-P. Effects of modafinil on the sleep EEG depend on Val158Met genotype of COMT. Sleep. 2010;33:1027-35.

17. Goel N, Uddin M, Banks S, Lin L, Mignot E, Dinges DF. Catechol-O-methyltransferase Val158Met polymorphism associates with individual differences in sleep physiologic responses to chronic sleep loss. PLoS ONE. 2011;6:e29283.

18. Borbély AA. A two process model of sleep regulation. Hum Neurobiol. 1982;1:195-204

19. Hefti K, Holst SC, Sovago J, Bachmann V, Buck A, Ametamey SM, et al. Increased metabotropic glutamate receptor subtype 5 availability in human brain after one night without sleep. Biol Psychiatry. 2013;73:161-8.

20. Holst SC, Bersagliere A, Bachmann V, Berger W, Achermann P, Landolt H-P. Dopaminergic role in regulating neurophysiological markers of sleep homeostasis in humans. J Neurosci. 2014;34:566-73.

21. Xie T, Ho SL, Ramsden D. Characterization and implications of estrogenic downregulation of human catechol-O-methyltransferase gene transcription. Mol Pharmacol. 1999;56:31-38.

22. Dauvilliers $Y$, Neidhart E, Tafti M. Sexual dimorphism of the catechol-O-methyltransferase gene in narcolepsy is associated with response to modafinil. Pharm J. 2002;2:65-68.

23. Gogos JA, Morgan M, Luine V, Santha M, Ogawa S, Pfaff D, et al. Catechol-Omethyltransferase-deficient mice exhibit sexually dimorphic changes in catecholamine levels and behavior. Proc Natl Acad Sci USA. 1998;95:9991-6.

24. Basner M, Dinges DF. Maximizing sensitivity of the psychomotor vigilance test (PVT) to sleep loss. Sleep. 2011;34:581-91.

25. McNair DM, Lorr M, Droppelman LF. EdITS Manual for the profile of mood states (POMS). San Diego, CA, USA: Educational and Industrial Testing Service; 1992.

26. Schulz H, Volk S, Yassouridis A. Measuring tiredness by symptoms. Sleep Res A. 1991;20:515.

27. Åkerstedt T, Gillberg M. Subjective and objective sleepiness in the active individual. Int J Neurosci. 1990;52:29-37.

28. Rechtschaffen A, Kales A. A manual of standardized techniques and scoring system for sleep stages of human subjects. Washington, DC USA: Gov Print Off; 1968.

29. Feinberg I, Floyd TC. Systematic trends across the night in human sleep cycles. Psychophysiology. 1979;16:283-91.

30. Napolitano A, Zürcher G, Prada M, Da. Effects of tolcapone, a novel catechol-Omethyltransferase inhibitor, on striatal metabolism of I-DOPA and dopamine in rats. Eur J Pharmacol. 1995;273:215-21.

31. Rojo A, Fontán A, Mena MA, Herranz A, Casado S, Yébenes JGde. Tolcapone increases plasma catecholamine levels in patients with Parkinson's disease. Park Relat Disord. 2001;7:93-96.

32. Dingemanse J, Jorga K, Zürcher G, Schmitt M, Sedek G, Da Prada M, Van Brummelen P. Pharmacokinetic-pharmacodynamic interaction between the COMT inhibitor tolcapone and single-dose levodopa. $\mathrm{Br} J$ Clin Pharmacol. 1995;40:253-62.

33. Holst SC, Müller T, Valomon A, Seebauer B, Berger W, Landolt H-P. Functional polymorphisms in dopaminergic genes modulate neurobehavioral and neurophysiological consequences of sleep deprivation. Sci Rep. 2017;7:45982.

34. Cohen J. Statistical power analysis for the behavioral sciences. New York, NY: Academic Press; 2013.

35. Richardson JTE. Eta squared and partial eta squared as measures of effect size in educational research. Educ Res Rev. 2011;6:135-47.

36. Gasparini M, Fabrizio E, Bonifati V, Meco G. Cognitive improvement during tolcapone treatment in Parkinson's disease. J Neural Transm. 1997;104:931-41.
37. Risbrough V, Ji B, Hauger R, Zhou X. Generation and characterization of humanized mice carrying COMT158 Met/Nal alleles. Neuropsychopharmacology. 2014;39:1823-32.

38. Tomlinson A, Grayson B, Marsh S, Hayward A, Marshall KM, Neill JC. Putative therapeutic targets for symptom subtypes of adult ADHD: D4 receptor agonism and COMT inhibition improve attention and response inhibition in a novel translational animal model. Eur Neuropsychopharmacol. 2015;25:454-67.

39. Lim J, Dinges DF. Sleep deprivation and vigilant attention. Ann NY Acad Sci. 2008;1129:305-22.

40. Ma N, Dinges DF, Basner M, Rao H. How acute total sleep loss affects the attending brain: a meta-analysis of neuroimaging studies. Sleep. 2015;38: 233-40.

41. Langner R, Eickhoff SB. Sustaining attention to simple tasks: a meta-analytic review of the neural mechanisms of vigilant attention. Psychol Bull. 2013;139:870-900.

42. Haber SN. The place of dopamine in the cortico-basal ganglia circuit. Neuroscience. 2014;282:248-57.

43. Käenmäki $M$, Tammimäki $A$, Myöhänen $T$, Pakarinen $K$, Amberg $C$, Karayiorgou $M$, et al. Quantitative role of COMT in dopamine clearance in the prefrontal cortex of freely moving mice. J Neurochem. 2010;114:1745-55.

44. Sesack SR. Prefrontal cortical dopamine transmission: ultrastructural studies and their functional implications. Synapse Struct Funct. 2014;467-501. http://www. sciencedirect.com/science/article/pii/B9780124186750000158.

45. Chuah LYM, Chee MWL. Cholinergic augmentation modulates visual task performance in sleep-deprived young adults. J Neurosci. 2008;28:11369-77.

46. Chuah LYM, Chong DL, Chen AK, Rekshan WR, Tan J-C, Zheng $H$, et al. Donepezil improves episodic memory in young individuals vulnerable to the effects of sleep deprivation. Sleep. 2009;32:999-1010.

47. Andersen ML, Martins PJF, D'Almeida V, Bignotto M, Tufik S. Endocrinological and catecholaminergic alterations during sleep deprivation and recovery in male rats. J Sleep Res. 2005;14:83-90.

48. Léna I, Parrot S, Deschaux O, Muffat-Joly S, Sauvinet V, Renaud B, et al. Variations in extracellular levels of dopamine, noradrenaline, glutamate, and aspartate across the sleep-wake cycle in the medial prefrontal cortex and nucleus accumbens of freely moving rats. J Neurosci Res. 2005;81:891-9.

49. McMorris T, Harris RC, Swain J, Corbett J, Collard K, Dyson RJ, et al. Effect of creatine supplementation and sleep deprivation, with mild exercise, on cognitive and psychomotor performance, mood state, and plasma concentrations of catecholamines and cortisol. Psychopharmacology (Berl). 2006;185:93-103.

50. Eban-Rothschild A, Rothschild G, Giardino WJ, Jones JR, Lecea Lde. VTA dopaminergic neurons regulate ethologically relevant sleep-wake behaviors. Nat Neurosci. 2016;19:1356-66.

51. Andretic R, Andretic R, Franken P, Franken P, Tafti M, Tafti M. Genetics of sleep. Annu Rev Genet. 2008;42:361-88.

52. Oishi Y, Suzuki Y, Takahashi K, Yonezawa T, Kanda T, Takata Y, et al. Activation of ventral tegmental area dopamine neurons produces wakefulness through dopamine D2-like receptors in mice. Brain Struct Funct. 2017. https://doi.org/ 10.1007/s00429-017-1365-7.

53. Ueno T, Tomita J, Tanimoto $H$, Endo $K$, Ito $K$, Kume $S$, et al. Identification of a dopamine pathway that regulates sleep and arousal in Drosophila. Nat Neurosci. 2012;15:1516-23.

54. Volkow ND, Tomasi D, Wang G-J, Telang F, Fowler JS, Logan J, et al. Evidence that sleep deprivation downregulates dopamine D2R in ventral striatum in the human brain. J Neurosci. 2012;32:6711-7.

55. Wisor JP, Nishino S, Sora I, Uhl GH, Mignot E, Edgar DM. Dopaminergic role in stimulant-induced wakefulness. J Neurosci. 2001;21:1787-94.

56. Dauvilliers $Y$, Neidhart E, Lecendreux M, Billiard M, Tafti M. MAO-A and COMT polymorphisms and gene effects in narcolepsy. Mol Psychiatry. 2001;6: 367-72.

57. Jawinski P, Tegelkamp S, Sander C, Hantzsch M, Huang J, Mauche N, et al. Time to wake up: no impact of COMT Val158Met gene variation on circadian preferences, arousal regulation and sleep. Chronobiol Int. 2016;33:893-905.

58. Valomon A, Holst SC, Bachmann V, Viola AUAU, Schmidt C, Zürcher J, et al. Genetic polymorphisms of DAT1 and COMT differentially associate with actigraphy-derived sleep-wake cycles in young adults. Chronobiol Int. 2014;31:705-14.

59. Landolt HP, Raimo EB, Schnierow BJ, Kelsoe JR, Rapaport MH, Gillin JC. Sleep and sleep electroencephalogram in depressed patients treated with phenelzine. Arch Gen Psychiatry. 2001;58:268-76.

60. Smith ME, Farah MJ. Are prescription stimulants "smart pills"? The epidemiology and cognitive neuroscience of prescription stimulant use by normal healthy individuals. Psychol Bull. 2011;137:717-41.

61. Johns MW. A new method for measuring daytime sleepiness: the Epworth sleepiness scale. Sleep. 1991;14:540-5.

62. Wirz-Justice A, Roenneberg T, Merrow M. Life between clocks: daily temporal patterns of human chronotypes. J Biol Rhythm. 2003;18:80-90. 
Sleep deprivation, tolcapone, and sustained attention...

A Valomon et al.

63. Spielberger CD, Gorsuch RL, Lushene RE. Manual for the State-Trait Anxiety Inventory. Palo Alto, CA, USA: Consult Psychol Press; 1970. p. 638-42.

64. Buysse DJ, Reynolds CF,III, Monk TH, Berman SR, Kupfer DJ. The Pittsburgh Sleep Quality Index: a new instrument for psychiatric practice and research. Psychiatry Res. 1989;28:193-213.

65. Ruch W. Die revidierte Fassung des Eysenck Personality Questionnaire und die Konstruktion des deutschen EPQ-R bzw. EPQ-RK. Z Differ Diagn Psychol. 1999;20:1-24.
66. Galliaud E, Taillard J, Sagaspe P, Valtat C, Bioulac B, Philip P. Sharp and sleepy: evidence for dissociation between sleep pressure and nocturnal performance. J Sleep Res. 2008;17:11-5.

67. Leproult R, Colecchia EF, Berardi AM, Stickgold R, Kosslyn SM, Van Cauter E. Individual differences in subjective and objective alertness during sleep deprivation are stable and unrelated. Am J Physiol Reg Integ Comp Physiol. 2003;284: R280-90 